STUDI

FRANCESI

\section{Studi Francesi}

Rivista quadrimestrale fondata da Franco Simone

145 (XLIX | I) | 2005

Varia - fasc. I - gennaio-aprile 2005

\title{
Mary Gallagher, Soundings in French Caribbean Writing Since 1950. The shock of space and time
}

\section{Paola Ghinelli}

\section{(2) OpenEdition}

1 Journals

\section{Édition électronique}

URL : http://journals.openedition.org/studifrancesi/36822

DOI : $10.4000 /$ studifrancesi.36822

ISSN : 2421-5856

Éditeur

Rosenberg \& Sellier

\section{Édition imprimée}

Date de publication : 1 juillet 2005

Pagination : 208-209

ISSN : 0039-2944

\section{Référence électronique}

Paola Ghinelli, « Mary Gallagher, Soundings in French Caribbean Writing Since 1950. The shock of space and time », Studi Francesi [En ligne], 145 (XLIX | I) | 2005, mis en ligne le 30 novembre 2015, consulté le 18 avril 2021. URL : http://journals.openedition.org/studifrancesi/36822 ; DOI : https://doi.org/ 10.4000/studifrancesi.36822

Ce document a été généré automatiquement le 18 avril 2021.

\section{(c) $(1) \odot$}

Studi Francesi è distribuita con Licenza Creative Commons Attribuzione - Non commerciale - Non opere derivate 4.0 Internazionale. 


\title{
Mary Gallagher, Soundings in French Caribbean Writing Since 1950. The shock of space and time
}

\author{
Paola Ghinelli
}

\section{RÉFÉRENCE}

MARY GALLAGHER, Soundings in French Caribbean Writing Since 1950. The shock of space and time, Oxford, Oxford University Press, 2002.

1 La thèse principale de cet ouvrage est que l'intersection entre l'espace et le temps est fondamentale pour les romans caribéens de langue française; en effet, les exemples fournis tout au long des chapitres sont convaincants. Depuis l'introduction, l'essayiste met en relation les romans de son corpus avec d'autres œuvres caribéennes non francophones sur la base d'observations de critiques et d'experts de la Caraibe tels Michael Dash ou Antonio Benìtez-Rojo. La structure de l'essai est claire : la première partie se concentre sur le temps, la dernière sur l'espace, et elles sont séparées par un chapitre qui analyse l'intersection de ces deux dimensions dans le roman caribéen contemporain. La première partie donne aussi l'occasion à l'auteur de s'interroger sur l'ampleur du discours théorique des écrivains martiniquais par rapport à leurs homologues guadeloupéens. En effet, les trois chapitres consacrés au temps pivotent autour d'une interprétation évolutionniste de la succession négritude-antillanitécréolité, des mouvements littéraires dont les rapports avec l'espace et surtout avec le temps sont synthétisés en succession. C'est le mouvement de la Créolité qui attire surtout l'attention de Mary Gallagher, qui en montre les contradictions et les limites, mais qui semble le considérer comme la théorisation la plus pertinente avec son corpus.

Plusieurs romans sont cités pour montrer que le temps séquentiel est refusé dans la littérature caribéenne, qu'il est projeté sur l'espace, et que cette projection crée une 
relation complexe entre les narrations et l'histoire. Cette observation permet à Gallagher de remarquer l'absence d'intrigue ou mieux, la circularité ouverte de la temporalité littéraire caribéenne. À son avis, c'est l'éloignement (non seulement temporel mais aussi spatial) du passé qui crée une spécificité littéraire caribéenne et qui met à l'épreuve les notions de temps, d'histoire et de mémoire. C'est justement avec une réflexion sur la notion de mémoire et sur celle d'identité que se conclut la première partie de l'essai. Selon l'auteur la mémoire, celle collective en particulier, est plus présente dans la littérature caribéenne contemporaine que l'histoire elle-même. Encore une fois, l'essai démontre que le rapport avec le temps est crucial, parce que, selon Chamoiseau en particulier, l'espace est la trace du temps, donc la mémoire. D'autres auteurs, comme Pineau ou Condé, identifient les mécanismes de la mémoire avec le déplacement. La fréquence du récit d'enfance dans le corpus est aussi mentionnée dans ce chapitre. Mary Gallagher suppose que ce genre est privilégié parce que la distance temporelle de l'enfance permet aux auteurs de la narrer comme s'il s'agissait d'une île hors du temps.

3 Le chapitre qui forme le lien entre la partie consacrée au temps et celle consacrée à l'espace, analyse l'intertextualité en tant qu'acte lié à la tradition littéraire et à la mémoire. L'intertextualité est lue ici comme une modalité de la relation, une relation absolue qui concerne aussi les lieux et les temps. L'espace est le centre d'intérêt de la deuxième moitié de l'ouvrage, qui s'articule en une étude de l'habitation en tant que forme structurelle de l'imaginaire, en un chapitre sur le rapport entre ville et espace rural, et en une section consacrée aux liens que la culture franco-caribéenne possède avec des espaces extérieurs, notamment la France métropolitaine et l'Afrique. La plantation et l'espace urbain sont considérés les espaces principaux de la littérature caribéenne contemporaine. Encore une fois, la dimension spatiale est indissociable de la dimension temporelle, parce que la plantation est définie le lieu de mémoire par excellence dans le contexte littéraire, à cause de sa compromission avec le système esclavagiste. L'analyse de l'habitation en tant qu'espace étudie l'évolution de la représentation de la résistance au fil des cinquante dernières années de littérature antillaise. La réflexion sur le rôle littéraire du lieu urbain suit le chapitre consacré à l'habitation. L'auteur maintient la structure vaguement chronologique de son étude, mais le choix d'analyser l'espace urbain, fondamental pour la littérature antillaise, après avoir affronté la dimension temporelle et l'autre espace essentiel pour la structure des romans, permet à Mary Gallagher d'appuyer son analyse sur les hypothèses qu'elle a vérifiées préalablement, et donc de l'enrichir. Les deux derniers espaces dont parle l'essayiste sont ailleurs que dans l'archipel caraïbe. Le rapport conflictuel et parfois aliénant des départements d'outre-mer avec la France métropolitaine est parcouru au fil des pages des romans. L'auteur se demande si l'ironie, dont la littérature des dernières années semble se servir pour narrer ce rapport, pourra dépasser les modalités néo-coloniales de la vie quotidienne antillaise ou si elle déplace plutôt ces modalités au niveau de la réception. Cette alternative montre, à mon avis, un des défauts de cet essai, qu'il est possible de retrouver ailleurs. L'auteur passe parfois sans transition d'une perspective interne à la littérature à une perspective externe. Par exemple, elle met parfois sur le même plan les citations tirées des œuvres de son corpus et celles tirée des interviews que leurs auteurs ont accordées, alors que ce passage de l'analyse proprement littéraire à celle des mécanismes de diffusion et de réception devrait être mieux marqué. Il aurait peut-être été nécessaire de consacrer un chapitre à une focalisation externe et à l'étude du rôle de la littérature 
caribéenne francophone dans le marché éditorial. Si le rapport avec l'Afrique exclut la domination directe, il n'en est pas pour autant moins compliqué. Gallagher en effet démontre qu'une relation directe et simultanée à l'Afrique est impossible pour la littérature en objet. Le dernier chapitre est parmi les plus intéressants de cet ouvrage, parce qu'il essaye d'établir une relation entre une conception spatio-temporelle contemporaine, affectée par les processus de globalisation, et la littérature antillaise francophone. Soundings in French caribbean writing since 1950 est une étude nécessaire, avec un corpus très vaste et une perspective critique qui dépasse efficacement les limites de la francophonie. Ces quelques conditions préalables auraient pu pousser Mary Gallagher encore plus loin, en lui permettant d'élaborer un travail de synthèse plus complet en conclusion d'une analyse aussi articulée. Néanmoins, les observations que l'auteur fait dans cet essai restent précieuses, ainsi que son ouverture critique à des essais et à des œuvres qui se situent en dehors du champ littéraire caribéen francophone habituellement considéré par les critiques, mais qui ne sont pas pour autant moins pertinentes avec le sujet de l'essai. 